$\xi=$ 잘

\title{
Dentigerous cyst of maxilla and mandible associated with ectopic teeth- a case report
}

\author{
Geeta Singh ${ }^{1}$, Dichen Palmo Bhutia ${ }^{2 *}$, Diksha Singh ${ }^{3}$, Jagdish Gamit ${ }^{4}$, U. Vignesh ${ }^{5}$ \\ ${ }^{1}$ Associate Professor, Department of Oral and Maxillofacial Surgery, King George's Medical University, \\ Lucknow, Uttar Pradesh, India \\ ${ }^{2}$ Senior Resident, Department of Oral and Maxillofacial Surgery, King George's Medical University, Lucknow, Uttar Pradesh, India \\ 3 Assistant Professor, Department Oral Pathology, King George's Medical University, Lucknow, Uttar Pradesh, India \\ ${ }^{4}$ Department of Oral and Maxillofacial Surgery, , King George's Medical University, Lucknow, Uttar Pradesh, India \\ ${ }^{5}$ Junior Resident, Department of Oral and Maxillofacial Surgery, King George's Medical University, Lucknow, Uttar Pradesh ,India \\ *Corresponding author E-mail: dichen.namchang@gmail.com
}

\begin{abstract}
Background: Dentigerous cyst (DC) is the second most common cyst of the jaw after radicular, affecting mostly the permanent dentition in the mandible (70\%). DC Of maxillary sinus involving ectopic teeth is rare.

Case details: We report a case of DC of maxillary sinus as well as anterior mandible associated with ectopic teeth, in a non-syndromic 8 years old boy who underwent cyst enucleation along with extraction of the involved teeth.

Conclusion: DC affecting both maxilla and mandible is a less common entity. Treatment modality can range from enucleation to simple marsupilisation depending upon the extent of the lesion.
\end{abstract}

Keywords: Dentigerous Cyst; Maxillary Sinus; Enucleation; Ectopic Teeth

\section{Introduction}

Dentigerous cyst (DC), also termed as "follicular cyst"are the most common type of developmental odontogenic cyst associated with impacted, unerupted, or embedded teeth Prashad et al. 2007, Buyukkurt et al. 2010) .Multiple DC generally occur in association with a developmental syndrome or systemic diseases. Abnormal tissue interaction during tooth development may potentially result in ectopic tooth development and eruption (Prashad et al. 2007, Buyukkurt el al. 2010). The pathogenesis of the cyst is unknown and are believed to originate from dental follicles. The epithelial -lined developmental cavity encloses the crown of an unerupted tooth at the cement-enamel junction (Ko el al. 1999). The expansion of dental follicles resulting from accumulation of fluid between reduced enamel epithelium and tooth enamel exerts pressure on the follicle which in turn obstructs the venous outflow leading to rapid transduction of serum across capillary walls increasing the hydrostatic pressure (Ziccardi el al. 1997). This separates the follicle from the crown. DC account for about $24 \%$ of all jaw cyst ( Ko el al. 1999). Third molars, maxillary canines and premolars are the most frequently affected teeth. Maxillary teeth are often displaced into the maxillary sinus and hence, the sequelae may vary from sinus obstruction to even blindness (Litvin et al. 2008).

DC can be treated by either enucleation or marsupilisation . Enucleation of the cyst along with extraction of the associated unerupted teeth. However, enucleation is considered more aggressive whereas marsupilization comes with the disadvantage of recurrence or persistence of the lesion (Tournas et al. 2006, Buyukkurt et al. 2010,). In larger cyst initial marsupilization to diminish the osseous defect, followed by enucleation and extraction has been advocate (Ziccardi el al. 1997, Buyukkurt et al. 2010,).

In this paper we report a case of DC in a 8 year old boy, associated with unerupted teeth in the maxillary sinus and anterior mandible.

\section{Case report}

A 8 year old boy visited our Out -Patient Department with a chief complaint of swelling over left side face for the last 2 months. On examination a swelling measuring approximately $4 \times 3 \mathrm{cms}$ was found on left side of the face extending from left infra-ortital rim upto the left corner of the mouth (Fig 4). Mediolaterally, the swelling extended from left ala of the nose, mildly elevating the nasobuccal crease, upto lateral canthus of left eye. Intraoral examination showed swelling over left alveolobuccal complex, obliterating the vestibule from left lateral maxillary incisor upto the tuborisity. Labio-palatal expansion was noted in the anterior arch of maxilla causing gross mobility of the erupting first and second bicuspid. Intraoral mandibular finding revealed mild degree of vestibule elevation in the region of left lateral incisor region, which was displaced. On palpation, the swellings were non-tender, semisolid and fluctuant. 


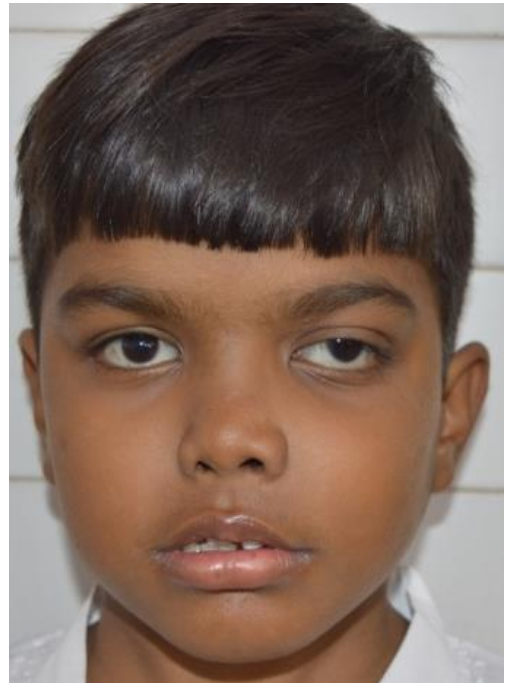

Fig. 1: Extra-Oral Photograph Showing Diffuse Swelling Over Left Side Of Maxilla.

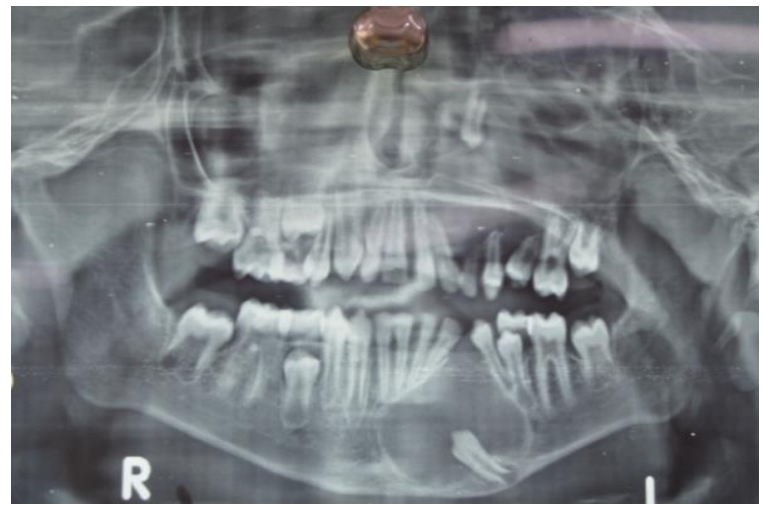

Fig. 2: OPG Showing Cysts with Impacted Teeth in Maxilla and Mandible.

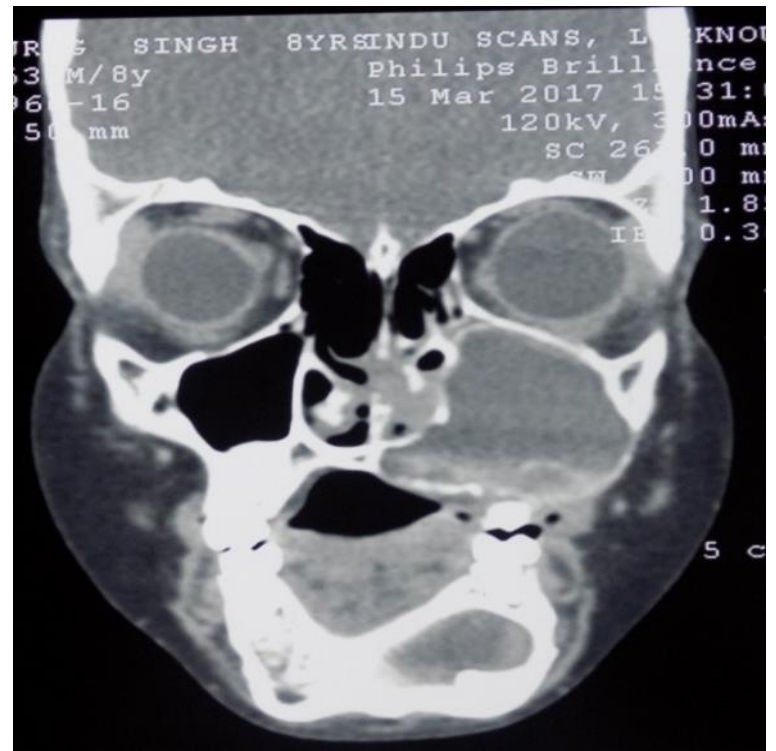

Fig. 3: Coronal CT Section Showing Expansion of Left Maxillary Sinus with Erosion of Left Lateral Wall of Nasal Cavity Along With Radiolucency in Mandible.

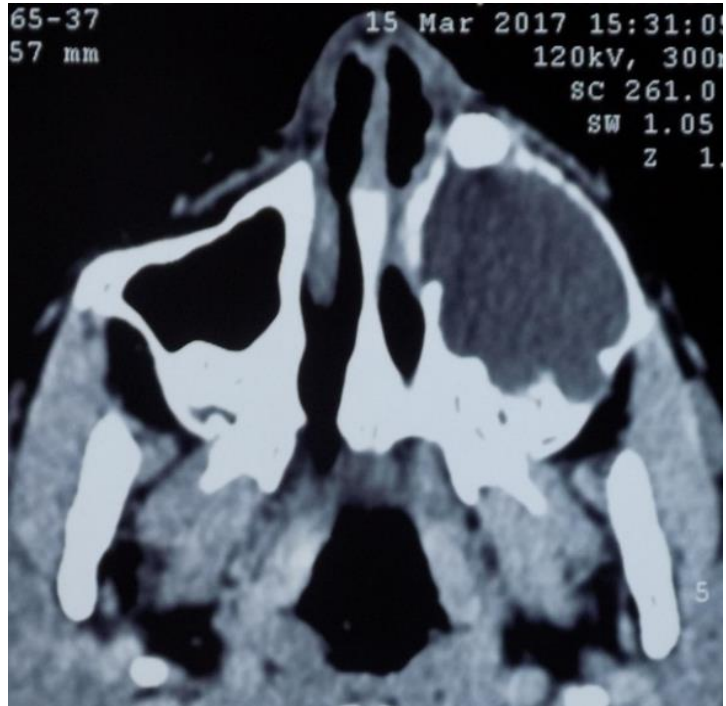

Fig. 4: Axial CT Section Showing Expansion of Left Maxillary Sinus with Impacted Canine Located Anteromedially.

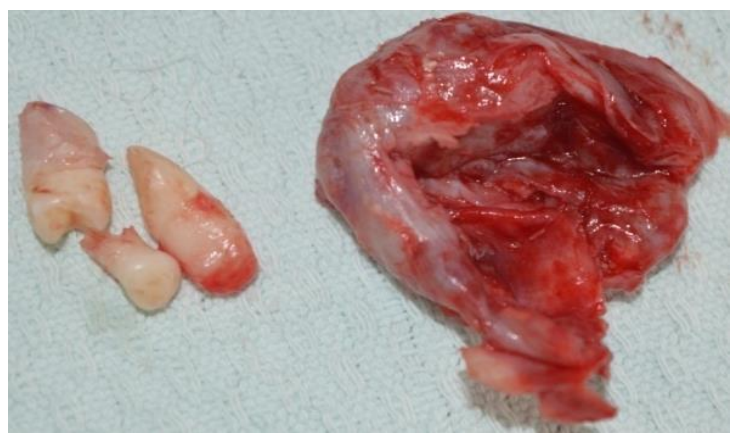

Fig. 5: Specimen of Enucleated Cystic Wall with Extracted Teeth.

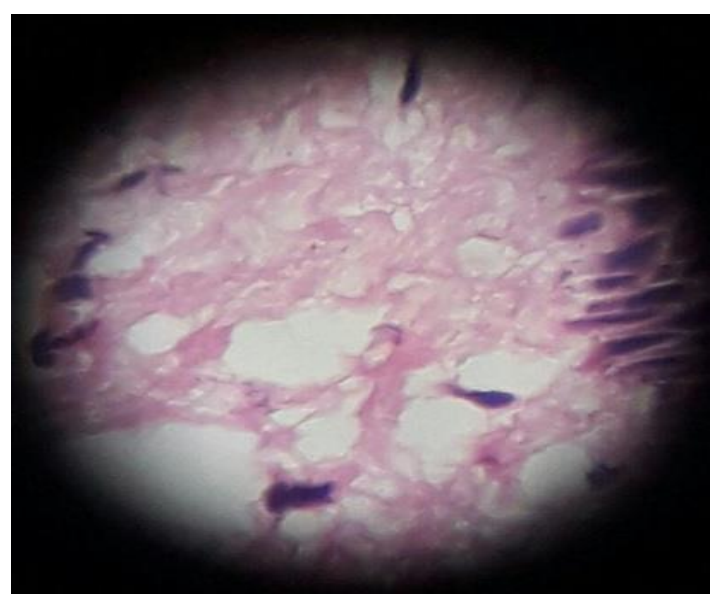

Fig. 6: H\&E Stained Slide (10x) Suggestive Lining of Stratified Squamous Epithelium and Granulation Tissue with Chronic Inflammatory Infiltrate.

Panaromic radiograph revealed a relatively large and well- defined radiolucency on the left side of maxilla as well as anterior mandible, both enveloping an unerupted teeth. The unerupted teeth were displaced anterosuperiorly in maxilla and inferiorly near the lower border in the mandible (Fig.2). Computed Tomography scan showed a cystic lesion measuring $4.5 \mathrm{cms}$ horizontally, $4 \mathrm{cms}$ vertically and $3 \mathrm{cms}$ antero-posteriorly with expansion and erosion of maxillary sinus cortical bone. (Fig: 3 and 4). The mass was seen to extend into left lateral nasal wall displacing the osteo-meatal complex towards the opposite side and causing mild elevation of the orbital floor. Posterioly the mass was seen extending upto left pterygomaxillary fissure (Fig. 3 and 4). The cyst in the mandible showed mild intra -bony expansion measuring $4 \mathrm{cms} \times 2 \mathrm{cms} \times$ $1 \mathrm{cms}$ along with erosion of buccal cortical plate in the anterior mandible without involving the lingual cortex. The tooth con- 
tained in the cyst was displaced inferiorly towards the lower border.

After haematological analysis the patient underwent enucleation of the cysts with extraction of the impacted teeth under General Anesthesia. Caldwell -Luc approach was employed to access the cyst in the maxillary sinus .The cystic lining was identified after breaching into the thinned antero and posterolateral wall and was separated from the sinus walls underneath. A maxillary impacted canine was found near the anteromedial wall. Careful dissection lead to removal of whole of the cystic lining in-toto along with the impacted canine (Fig.5). Vestibular incision was used to enucleate the mandibular cyst in-toto along with the embedded tooth. Labelled samples from maxilla and mandible in two different specicans were sent for histopathology examination.

Histopathological examination revealed a lining of stratified squamous epithelium and granulation tissue with chronic inflammatory infiltrate in the underlying connective tissue. Occasional mature keratinizing squamous cell nests was noted in the collagenous cystic wall (Fig.6). A diagnosis of dentigerous cyst was made for both the samples.

\section{Discussion}

Dentigerous cysts are the most common type of developmental odontogenic cyst and are the second most common cystic lesion of the jaw, after radicular cyst (Tournas et al. 2006, Buyukkurt et al. 2010). About $70 \%$ of the cyst occur in posterior mandible and 30 $\%$ in maxilla. It usually involves the third molars .In our case, the cyst was found in the mandible as well as the maxilla involving the unerupted cuspids. Moreover,the cyst in the mandible was found in the anterior region, in contrary to its most common location.

DC are usually present in the second or third decade of life and rarely seen in childhood (O'Neil et al. 1989, Ustunner et al. 2003). Few authors suggest its prevelance among the younger patients below 20 yrs old (Takagi \& Koyoma 1999, Ertas \& Selim 2003,). However,age range of 4 yrs to $57 \mathrm{yrs}$ are reported. In our case it was seen to be affecting a eight yrs old boy.

DC are known be to asymptomatic which gradually grows over time and are generally presented only when it attains sizes big enough to cause gross intraoral swelling or teeth displacements and facial asymmetries while others are found incidentally during routine radiographic examinations(Tournas et al. 2006). In our case, the patient presented to us with swelling over his left side of mid-face with a duration of just one month while he had no clinical presentation of the cyst in the mandible, which was discovered in radiographs that were only intended to be taken for the maxillary cyst. Cyst involving the maxillary sinus may be presented with symptoms like swelling, pain,headache,and nasolacrimal obstruction (Takagi \& Koyoma 1999 , Tournas et al. 2006). Ophthalmic symptoms like epiphora, proptosis, diplopia (Golden et al. 1981, Atlas et al. 1997) and even blindness have been reported (Savundranayagam 1972). Numbness or parasthesia due to nerve compression can also occur (Takagi \& Koyoma 1999).

Water view, OPG, plain skull radiographs are simple and inexpensive radiographs that can be utilized to detect cystic lesions of jaws. Panaromic views are favoured over CT by some as to detect the structures of teeth. However, CT scans are requisite in defining the extension of masses extending into surrounding vital structures like the nasal or orbital cavity. Also they provide superior detail on bony involvement, associated pathology, exact location of ectopic tooth all of which help in definite diagnosis and treatment planning( Atlas et al. 1997).

Differetial diagnosis of DC includes radicular cyst, odontogenic cyst, odontogenic keratocyst,odontogenic tumours like ameloblastoma, Pindborg tumour, odontoma. However, mucoceles, retention cyst and pseudocyst can be included in the differential diagnosis in case of maxillary cyst (Ustunner et al. 2003, Tournas et al. 2006]. Odontogenis tumours like ameloblastoma or epidermoid carcinomas are reported to be risen from lining of DC (Ustunner et al.
2003, Tournas et al. 2006). No metaplastic and dysplastic changes arising from DC of maxillary sinus are reported in the literature. The Standard Treatment for DC is enucleation and extraction of the cyst-associated impacted or unerupted teeth (Tournas et al. 2006, Buyukkurt et al. 2010 9). However, marsupilization are advisable in larger cyst and thought to be more efficient in children (Ko el al. 1999, Takagi \& Koyoma 1999) as it conserves the affected tooth and facilitates its eruption. However, certain factors are requisite to determine the eruption potential of the involved tooth. Some studies shows that if the axis of inclination of the tooth less than $80^{\circ}$ of tooth axis angulation or less than $9 \mathrm{~mm}$ depth in bone are predictive of eruption(Hyomoto et al. 2003). In our case, the teeth involved were extracted along with complete cystic enucleation. The patient is being followed-up for the past 2 months with no evidence of recurrence till date.

\section{References}

[1] Buyukkurt MC, Omezli MM, Miloglu O. Dentigerous cyst associated with an ectopic tooth in the maxillary sinus: A report of 3 cases and review of the literature. Oral Surg Oral Med Oral Pathol Oral Radiol Endod. 2010; 109:67-71. [PubMed] https://doi.org/10.1016/j.tripleo.2009.07.043.

[2] Srinivasa Prasad T, Sujatha G, Niazi T, Rajesh P. Dentigerous cyst associated with an ectopic third molar in the maxillary sinus: A rare entity. Indian J Dent Res. 2007; 18:141-3. [PubMed] https://doi.org/10.4103/0970-9290.33793.

[3] Ko KS, Dover DG, Jordan RC. Bilateral dentigerous cysts-report of an unusual case and review of the literature. J Can Dent Assoc. 1999; 65(1):49-51. [PubMed]

[4] Ziccardi VB, Eggleston TI, Schneider RE. Using fenestration technique to treat a large dentigerous cyst. JADA. 1997; 128(2):201205. [PubMed] https://doi.org/10.14219/jada.archive.1997.0165.

[5] Litvin M, Caprice D, Infranco L. Dentigerous cyst of the maxilla with impacted tooth displaced into orbital rim and floor. Ear Nose Throat J. 2008; 87:160-2. [PubMed]

[6] Tournas A, Tewfik M, Chauvin P, Manoukian J. Multiple unilateral maxillary dentigerous cysts in a nonsyndromic patient: A case report and review of the literature. Int J Pediatr Otorhi Extra. 2006; 1:100-6 https://doi.org/10.1016/j.pedex.2005.12.005.

[7] Golden AL, Foote J, Lally E, Beideman R, Tatoian J. Dentigerous cyst of the maxillary sinus causing elevation of the orbital floor- report of a case. Oral Surg Oral Med Oral Pathol. 1981; 52:133-6. [PubMed] https://doi.org/10.1016/0030-4220(81)90308-X.

[8] O'Neil D, Mosby E, Lowe J. Bilateral mandibular dentigerous cysts in a five-year-old child: report of a case. ASDC J Dent Child. 1989; 56:382-4. [PubMed]

[9] Ustuner E, Fitoz S, Atasoy C, Erden I, Akyar S. Bilateral maxillary dentigerous cysts: A case report. Oral Surg Oral Med Oral Pathol Oral Radiol Endod. 2003; 95:632-5. [PubMed] https://doi.org/10.1067/moe.2003.123.

[10] Ertas U, Selim M. Interesting eruption of four teeth associated with a large dentigerous cyst in mandible by only marsupialization. J Oral Maxillofac Surg. 2003; 61(6):728-730. doi: 10.1053/joms.2003.50145.[PubMed] [Cross $\quad$ Ref] https://doi.org/10.1053/joms.2003.50145.

[11] Takagi S, Koyama S. Guided eruption of an impacted second premolar associated with a dentigerous cyst in the maxillary sinus of a 6 yr old child. J Oral Maxillofac Surg. 1999; 56:237-9. [PubMed] https://doi.org/10.1016/S0278-2391(98)90876-X.

[12] Atlas E, Karasen RM, Yilmamaz AB, Aktan B, Kocer I, Erman Z A case of large dentigerous cyst containing a canine tooth in the maxillary antrum leading to epiphora. J Laryngol Otol. 1997;111:641-3.[PubMed]

[13] Savundranayagam A. A migratory third molar erupting into the lower border of the orbit causing blindness in the left eye. Aust Dent J 1972; 17:418-20. https://doi.org/10.1111/j.18347819.1972.tb04970.x.

[14] Hyomoto M, Kawakami M, Inoue M, Kirita T: Clinical conditions for eruption of maxillary canines and mandibular premolars associated with dentigerous cysts. Am J Orthod Dentofacial Orthop 124:515, 2003. https://doi.org/10.1016/j.ajodo.2003.04.001. 